INPLASY

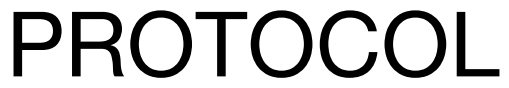

To cite: Zeng et al. Efficacy of Lycium barbarum $L$. on plasma lipid concentration in adults: A protocol for systematic review and meta-analysis. Inplasy protocol 2021110043. doi: 10.37766/inplasy2021.11.0043

Received: 13 November 2021

Published: 13 November 2021

Corresponding author: Jixiang Ren

renjx2003@163.com

Author Affiliation:

The Affiliated Hospital of Changchun University of

Chinese Medicine

Support: National Key R \& D Program.

Review Stage at time of this submission: Preliminary searches.

Conflicts of interest: None declared.

\section{Efficacy of Lycium barbarum L. on plasma lipid concentration in adults: A protocol for systematic review and meta-analysis}

Zeng, X1; Zhao, W2; Xu, Y3; Zhang, C4; Wu, J5; Xia, L6; Tian, Z7; Ren, J8.
Review question / Objective: Population: Patients were eligible for the study if they were adults(age $>18 \mathrm{y})$ populations of any sex; Intervention: Lycium barbarum L.; Compare: Comparisons interventions include placebo control, no therapy, exercise intervention, diet intervention, or active pharmacological treatment. Outcomes: effects of Lycium barbarum L. on the concentrations of total cholesterol (TC), triglyceride (TG), low density lipoprotein-cholesterol (LDL-C), and high density lipoprotein-cholesterol (HDL-C). Study: RCTs. Condition being studied: RCTS of Lycium barbarum L. on adults.

Information sources: Relevant studies were identified in the following electronic databases: China National Knowledge Infrastructure, PubMed, Cochrane Library, and Wanfang databases. $\mathbf{n}$ addition, a manual search of recent reviews, meta-analyses and original studies were also scrutinized.

INPLASY registration number: This protocol was registered with the International Platform of Registered Systematic Review and Meta-Analysis Protocols (INPLASY) on 13 November 2021 and was last updated on 13 November 2021 (registration number INPLASY2021110043).

\section{INTRODUCTION}

Review question / Objective: Population: Patients were eligible for the study if they were adults(age $>18 y$ ) populations of any sex; Intervention: Lycium barbarum L.;
Compare: Comparisons interventions include placebo control, no therapy, exercise intervention, diet intervention, or active pharmacological treatment. Outcomes: effects of Lycium barbarum L. on the concentrations of total cholesterol 
(TC), triglyceride (TG), low density lipoprotein-cholesterol (LDL-C), and high density lipoprotein-cholesterol (HDL-C). Study: RCTs.

Rationale: As a traditional herbal medicine and functional food, the dried ripe fruit of $L$. barbarum, also known as Goji berry or wolfberry, has been consumed for at least 2000 years in China. In support of these traditional properties, scientific evidence has shown that L. barbarum fruit possesses a variety of biological activities, such as anti-aging and increased metabolism. However, the results of supplemental L. barbarum on the plasma lipid concentration are also controversial. Therefore, this study will examine whether Lycium barbarum $L$. have benefits on plasma lipid concentration in adults.

Condition being studied: RCTS of Lycium barbarum $\mathrm{L}$. on adults.

\section{METHODS}

Search strategy: A systematic literature search was performed for publications from their starting dates through October 2021, with China National Knowledge Infrastructure, PubMed, Cochrane Library, and Wanfang databases. The search was restricted to published studies on humans without language restriction. We used the following keywords as search terms: ("Lycium barbarum L." OR "L. barbarum" OR " Goji" OR "lycium" OR" wolfberry" ) AND ("Intervention Studies" OR "intervention" OR "controlled trial" OR "randomized" OR "randomised" OR "random" OR "randomly" OR "placebo" OR "assignment"). In addition, a manual search of recent reviews, meta-analyses and original studies were also scrutinized.

Participant or population: Patients were eligible for the study if they were adults(age $>18 y$ ) populations of any sex.

Intervention: Lycium barbarum L.

Comparator: Comparisons interventions include placebo control, no therapy, exercise intervention, diet intervention, or active pharmacological treatment.

Study designs to be included: All relevant peer-reviewed articles with a randomized controlled trial (RCT) design will be included.

Eligibility criteria: RCTS of RCTS of Lycium barbarum $L$. on adults.

Information sources: Relevant studies were identified in the following electronic databases: China National Knowledge Infrastructure, PubMed, Cochrane Library, and Wanfang databases. $\mathbf{n}$ addition, $\mathbf{a}$ manual search of recent reviews, metaanalyses and original studies were also scrutinized.

Main outcome(s): Effects of Lycium barbarum $L$. on the concentrations of total cholesterol (TC), triglyceride (TG), low density lipoprotein-cholesterol (LDL-C), and high density lipoprotein-cholesterol (HDL-C).

Data management: Data for the trials will be extracted independently by two review authors using a standard form. The extracted information was as follows: (i) study characteristics (first author's last name, year of publication, location of the study, sample size and study design); (ii) participants' information (gender, mean age, mean body mass index [BMI], and health status); (iii) intervention details (duration of treatment and ginseng dose); and (iv) investigated outcomes including total cholesterol (TC), triglyceride (TG), LDL-C, and HDL-C.

Quality assessment / Risk of bias analysis: Each study included will be independently assesses by two independent reviewers. For any disagreement, a third reviewer will confirm the final assessment. Original authors will be contacted in the event of insufficient details to confidently assess the risk of bias. Based on the Cochrane risk of bias tool version 5.1.0 (updated March 2011), included articles will be rated as high, unclear or low for performance bias, attrition bias, and reporting bias on 
the following domains: 1) Random sequence generation (selection bias); 2) Allocation concealment (selection bias); 3) Blinding of participants and personnel (performance bias); 4) Blinding of outcome assessment (detection bias); 5) Incomplete outcome data (attrition bias); 6) Selective reporting (reporting bias); and 7) other bias. Studies will be classified as low risk or high risk categories.

Strategy of data synthesis: All RCTs data will be entered into a Rstudio software (Version 1.4.1717) to synthesize the evidence. Study characteristics will be summarized using: publication details (authors, publication year, location), sample size, sample characteristics, inclusion and exclusion criteria for participants, frequencies (\%) for categorical variables and mean (SD) for continuous variables. Any discrepancy that cannot be calculated from the paper will be requested from the corresponding author via email. All collected information and data will be organized and stored in Excel databases. If sufficient data are available and the studies/methods are sufficiently homogeneous regarding the interventions and outcomes, Meta-analysis will be performed using the software Rstudio version 1.4.1717 Statistical tests of heterogeneity will be carried out. Effect estimates will be weighted by the inverse of their variance, giving greater weight to larger trials. If it is not possible to conduct meta-analysis for any outcome due to insufficient data, a qualitative synthesis of the findings from the included studies will be provided, structured around the intervention type, population characteristics, outcome result and intervention content. To calculate effect sizes and $95 \%$ confidence intervals using random-effects models. Subgroup analyses for comparisons between TC and active and nonactive control interventions. In the case of missing data, original study authors will be contacted for confirmation. If data remain unavailable, a narrative description of these studies will be provided.

Subgroup analysis: If sufficient comparable studies are identified, we plan to conduct the following subgroup analysis. 1) Dosage and use time of wolfberry; 2) Comparison between different study populations (healthy orunhealthy).

Sensitivity analysis: In order to investigate the stability of the results, we will conduct a sensitivity analysis for the outcomes by omitting each of the RCT, or excluding the RCTs with high risk of bias, or excluding the RCTs with missing data.In this way, we will be able to assess the impact of individual studies on the overall results and whether the results are reliable. The conclusions will be compared and discussed according to the analysis results.

Language: We will only include studies published in English and Chinese.

Country(ies) involved: China.

Keywords: Lycium barbarum L., plasma lipid concentration, meta-analysis, protocol.

Contributions of each author:

Author 1 - Xueyuan Zeng.

Email: 760701754@qq.com

Author 2 - Weimin Zhao.

Email: 630123@163.com

Author 3 - Yunlong Xu.

Email: 16492111@qq.com

Author 4 - Chengwei Zhang.

Email: 1345282077@qq.com

Author 5 - Junliang Wu.

Email: w101900@foxmail.com

Author 6 - Libo Xia.

Email: 1875878987@qq.com

Author 7 - Ziyue Tian.

Email: 1765396676@qq.com

Author 8 - Jixiang Ren.

Email: renjx2003@163.com 